Nervenarzt 2009 $\cdot 80: 147-148$ DOI 10.1007/s00115-008-2643-7

Online publiziert: 23. Januar 2009

๑) Springer Medizin Verlag 2009

\author{
R. Hohlfeld \\ Institut für Klinische Neuroimmunologie, Klinikum der \\ LMU München, Campus Großhadern, München
}

\title{
B-Lymphozyten als neues "Target" der Multiple-Sklerose-Therapie
}

B-Lymphozyten und Antikörper sind seit einiger Zeit ins zentrale Blickfeld der MSForschung gerückt. Aktuelle Therapiestudien zielen auf die selektive Elimination bzw. Beeinflussung von B-Lymphozyten. $\mathrm{Zu}$ diesem aktuellen Thema erscheinen in dieser Ausgabe von „Der Nervenarzt“ zwei sehr informative Artikel von Schröder et al. (Rubrik Übersichten, S. 155) und Menge et al. (Rubrik Aktuelles, S. 190) [1, 2].

Welche genaue pathogenetische $\mathrm{Be}$ deutung den B-Zellen und Antikörpern bei der MS zukommt, ist noch weithin unbekannt [3]. Im Tiermodell der MS, der experimentell-autoimmunen Enzephalomyelitis (EAE), haben Antikörper lediglich eine „konditionale“ Rolle, d. h. sie wirken nur dann pathogen, wenn sie zusammen mit T-Lymphozyten appliziert werden. Dies liegt daran, dass peripher (z. B. intravenös) applizierte Antikörper die Blut-Hirn-Schranke nicht passieren können. Nur dann, wenn autoreaktive T-Lymphozyten zuvor die Blut-HirnSchranke durchlässig gemacht und eine Entzündung im Zentralnervensystem (ZNS) induziert haben, können Autoantikörper das ZNS erreichen und dort pathogene Effekte vermitteln.

Während die pathogene Rolle von $\mathrm{Au}$ toantikörpern noch ungeklärt ist, gibt es schon jetzt Hinweise dafür, dass Autoantikörper als diagnostische „Biomarker“ dienen können. Das überzeugendste Beispiel hierfür sind die kürzlich entdeckten Auto- antikörper gegen Aquaporin-4, die als diagnostische Marker der Neuromyelitis optica (NMO) große Bedeutung gewinnen [4]. Hingegen ist die Bedeutung von gegen andere ZNS-Antigene gerichteten $\mathrm{Au}$ toantikörpern weiterhin unklar.

Aus immunhistologischen Untersuchungen an Hirnbiopsiematerial ergeben sich Hinweise für eine immunpathologische Heterogenität der Multiplen Sklerose. So haben Lucchinetti, Brück und Lassmann eine antikörperdominierte Form der MS beschrieben, die sie als „Typ-II-MS“ bezeichnen [5]. Leider lassen sich die verschiedenen Formen der MS bisher nur histologisch identifizieren. Es erscheint plausibel, dass solche ,antikörperdominierten“ MS-Varianten besonders gut auf Therapien ansprechen, die selektiv gegen B-Lymphozyten gerichtet sind, wie zum Beispiel der monoklonale Anti-CD2o-Antikörper Rituximab [6]. Allerdings unterscheiden die laufenden Therapiestudien mangels verfügbarer Biomarker nicht zwischen den verschiedenen immunpathologischen Varianten der MS. Dennoch sind erste Studienergebnisse mit Rituximab vielversprechend [6].

Neben einer Reihe von neuen, selektiv die B-Lymphozyten beeinflussenden Therapien haben auch die bereits zugelassenen Therapien nachhaltige Effekte auf B-Lymphozyten. Neue Untersuchungen zeigen, dass Interferon $\beta$ den $B$ -
Zell-stimulierenden Faktor BAFF („B cell stimulating factor of the TNF family") induziert [7]. Daraus lässt sich ableiten, dass Interferon $\beta$ bei antikörperdominierten autoimmunen ZNS-Entzündungen, wie der Aquaporin-4-Antikörper-assoziierten NMO, eher ungünstig wirkt. Tatsächlich gibt es inzwischen eine ganze Reihe von Einzelfallbeobachtungen, die schwere Exazerbationen der NMO unter Interferontherapie berichten.

Eine weitere aktuelle Untersuchung derselben Arbeitsgruppe zeigte, dass auch Natalizumab Effekte auf B-Lymphozyten ausübt: Unter Therapie mit Natalizumab (Tysabri) steigt die Zahl der im Blut zirkulierenden B-Lymphozyten und besonders ihrer unreifen Vorläuferformen („Prä-BZellen") [8]. Wahrscheinlich werden BZellen und ihre Vorläufer einerseits an der Migration ins ZNS gehindert und andererseits vermehrt aus dem Knochenmark freigesetzt. Da vor allem B-Lymphozyten potenzielle Träger des JC-Virus sind, könnte dieser Mechanismus auch mit dem Risiko für die Entstehung einer progressiven multifokalen Leukoenzephalopathie von Tysabri zusammenhängen.

Die Autoren der Übersichtsartikel betonen zu Recht die Bedeutung von B-Lymphozyten als ein attraktives Ziel für zukünftige Therapiestrategien. Die noch bestehende Unkenntnis über die genaue pathogenetische Rolle der B-Lymphozyten, und das Risiko neuer Nebenwirkungen, 
dämpfen allerdings in diesem frühen Stadium allzu übertriebene Erwartungen.

\section{Korrespondenzadresse}

\section{R. Hohlfeld}

Institut für Klinische Neuroim-

munologie, Klinikum der LMU

München, Campus Großhadern

Marchioninistraße 15, 81377 München

reinhard.hohlfeld@med.uni-muenchen.de

\section{Literatur}

1. Menge $\mathrm{T}$, Büdingen $\mathrm{H}-\mathrm{C}$ von, Dalakas $\mathrm{MC}$ et al (2009) B-Zell-gerichtete Therapie in der Multiplen Sklerose - Aktueller Stand. Nervenarzt

2. Schröder A, Ellrichmann G, Chehab G et al (2009) Einsatz von Rituximab in der Behandlung neuroimmunologischer Erkrankungen. Nerverarzt

3. Meinl E, Krumbholz M, Hohlfeld R (2006) B lineage cells in the inflammatory CNS environment: Migration, maintenance, local antibody production and therapeutic modulation. Ann Neurol 59:880-892

4. Wingerchuk DM, Lennon VA, Lucchinetti CF et al (2007) The spectrum of neuromyelitis optica. Lancet Neurol 6:805-815

5. Lassmann H, BrückW, Lucchinetti C (2001) Heterogeneity of multiple sclerosis pathogenesis: implications for diagnosis and therapy. Trends Mol Med 7:115-121

6. Hauser SL, Waubant E, Arnold DL et al (2008) B-cell depletion with rituximab in relapsing-remitting multiple sclerosis. N Engl J Med 358:676-688

7. Krumbholz M, Faber H, Steinmeyer F et al (2008) Interferon-b increases BAFF levels in multiple sclerosis: implications for B cell autoimmunity. Brain 131:1455-1463

8. Krumbholz M, Meinl I, Kumpfel T et al (2008) Natalizumab disproportionately increases circulating pre-B and B cells in multiple sclerosis. Neurology 71:1350-1354

\section{Ausschreibung des Studien- Stipendiums der Deutschen Migräne- und Kopfschmerz- gesellschaft (DMKG) 2009}

Die Deutsche Migräne- und Kopfschmerzgesellschaft fördert durch ihre Stipendien und Preise die Erforschung von Ursachen und möglichen Therapien von Kopfschmerzerkrankungen.

Im Jahr 2009 plant die Deutsche Migräneund Kopfschmerzgesellschaft erstmals ein Studien-Stipendium zu vergeben. Dieses Studien-Stipendium soll den/die Antragsteller(in) in die Lage versetzen, eine Studie zur nicht medikamentösen Prophylaxe von Kopfschmerz-Erkrankungen durchzuführen, bzw. eine erfolgreiche spätere Antragstellung bei Stiftungen oder nationalen Förder-Einrichtungen zu ermöglichen. Ausdrücklich sollen dabei Projekt-Vorschläge gefördert werden, die nicht primär im Interesse der pharmazeutischen Industrie sind und daher von dieser auch nicht gefördert werden. Die Stipendien-Anträge sollen sich an den Vorgaben der Deutschen Forschungsgemeinschaft orientieren (siehe http://www.dfg.de/) und das Stipendium der DMKG als Startfinanzierung für einen DFG-Antrag benutzen. Eine entsprechende Antragstellung, bzw. Durchführung erster Pilot-Untersuchungen soll mit einem Betrag von bis zu $€ 50.000$ gefördert werden.

Eine spätere Studiendurchführung wird gegebenenfalls darüber hinaus auch durch die administrative Hilfe von Seiten der DMKG unterstützt.

Das Präsidium entscheidet nach Empfehlung von drei externen Gutachtern, darüber hinaus behält sich das Präsidium vor, das Preisgeld bei mehreren förderwürdigen Projekten zu teilen. Vollständige Anträge mit Lebenslauf und Publikationsliste sind bis zum 15.07.2009 einzureichen beim Generalsekretariat der DMKG, Herrn Professor Peter Kropp, Institut für Medizinische Psychologie im Zentrum für Nervenheilkunde, Medizinische Fakultät der Universität Rostock, Gehlsheimer Straße 20,18147 Rostock.

Quelle: Professor Peter Kropp (Rostock).

\section{Mattiacum Preis}

Ausgeschrieben ist der Mattiacum Preis der Deutschen Gesellschaft für Neurologie für hervorragende wissenschaftliche Leistungen in Grundlagen- oder wissenschaftlicher Forschung auf dem Gebiet der Motoneuron-Erkrankungen.

Dotation: $10.000 €$ (der Preis kann auch geteilt werden)

Bewerbungen bis zum 30. Juni 2009 sollten neben der preiswürdigen Arbeit (Publikation, Habilitation, o.ä.), einen Lebenslauf und eine Auflistung der Originalpublikationen enthalten. Die Unterlagen sind in sechsfacher Ausfertigung an den Vorsitzenden des Preiskuratoriums, Herrn Prof. Dr. med. Heinz Reichmann, Direktor der Klinik und Poliklinik für Neurologie, Technische Universität Dresden, Fetscherstr.74, D-01307 Dresden zu richten. E-Mail: Heinz.Reichmann@uniklinikumdresden.de

Quelle: Deutsche Gesellschaft für Neurologie, www.dgn.de 